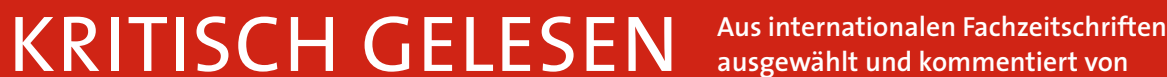

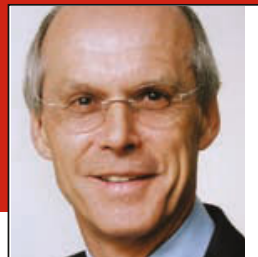

Prof. Dr. med. H. S. FüeßI

Isar-Amper-

Klinikum,

KI. München-

Ost, Haar

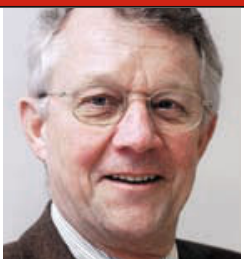

Prof. Dr. med. H. Holzgreve

Internist, Kardiologische Praxis, München

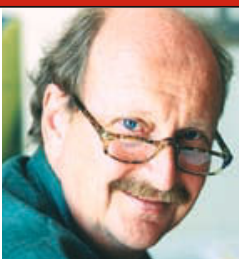

Prof. Dr. med.

E. Ernst

Peninsular

Medical School,

University

of Exeter/UK

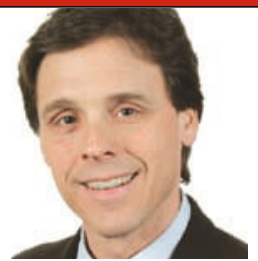

PD Dr. med. G. Nelles

Zentrum für Neurologie und Seelische Gesundheit, Köln

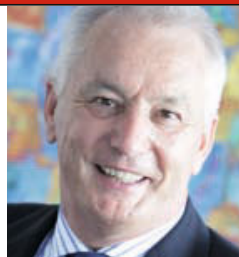

Prof. Dr. med. C. Diehm

SRH Klinikum Karlsbad-Langensteinbach

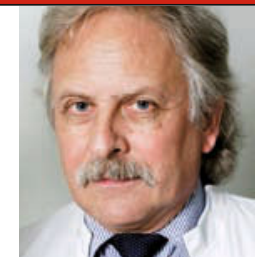

Prof. Dr. med. H.-C. Diener Neurologie, Universitätsklinik Essen

\section{Eigenverantwortung des Hypertonikers lohnt sich}

\section{Mit Blutdruckselbstmessung ein- schließlich Telemetrie und Selbst- medikation kann der Blutdruck von Hypertonikern besser eingestellt werden als durch die übliche Behandlung, allerdings ohne einen nennenswerten Rückgang der Praxisbesuche.}

- 264 unzureichend eingestellte Hypertoniker wurden wie üblich behandelt (Kontrollgruppe), 263 Patienten bestimmten zusätzlich zur medikamentösen Therapie ihren Blutdruck zweimal am Morgen für die Dauer einer Woche pro Monat (Interventionsgruppe). Per Tele-

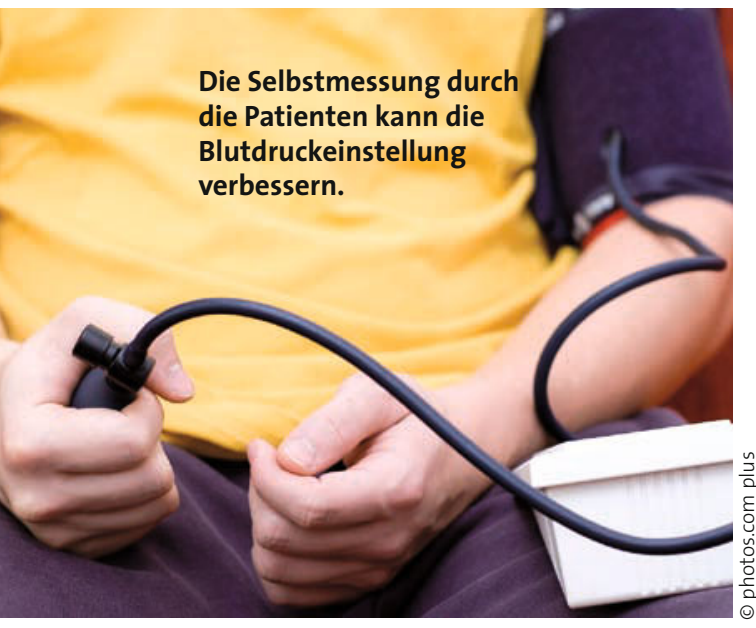

metrie wurden diese Werte an das Studienzentrum übermittelt.

Bei Werten über $130 / 85 \mathrm{mmHg}$ (Diabetiker über $130 / 75 \mathrm{mmHg}$ ) an vier oder mehr Tagen sollten die $\mathrm{Pa}$ tienten nach einem vorgegebenen Schema die Behandlung intensivieren. Nach zwei Intensivierungsschritten ohne Normalisierung des Blutdrucks erfolgte eine Vorstellung beim Hausarzt. Ferner waren die Patienten angehalten, bei Werten über 200/100 mmHg bzw. unter $100 \mathrm{mmHg}$ systolisch ihren Hausarzt zu kontaktieren. Wenn dies versäumt wurde, nahm das Forschungsteam telefonischen Kontakt auf.

Der Blutdruck sank in beiden Gruppen nach sechs Monaten um 12,9/5,2 mmHg und 9,2/3,9 mmHg bzw. nach zwölf Monaten um 17,6/7,5 mmHg und 12,2/4,8 mmHg. Die Blutdrucksenkung war also in der Interventionsgruppe um 3,7/1,3 mmHg bzw. 5,4/2,7 mmHg stärker als bei der üblichen Behandlung und bei der Einnahme von mehr Antihypertensiva.

Bei den Nebenwirkungen gab es keine Unterschiede, nur Beinödeme traten in der Interventionsgruppe, vermutlich wegen einer häufigeren bzw. höher dosierten Behandlung mit Kalziumantagonisten, öfter auf.

\section{Kommentar}

Die Autoren bezeichnen die Ergebnisse als „neue, bedeutende Bereicherung der antihypertensiven Therapie" in der Praxis. Bei der Bewertung muss man folgende Gesichtspunkte berücksichtigen: - Die erzielte Blutdrucksenkung mag auf den ersten Blick gering erscheinen, doch entspricht sie den Werten, die in den großen Interventionsstudien zu einem Rückgang von Schlaganfällen und kardialen Komplikationen führte. - In der Diabetesbehandlung und bei der Antikoagulation haben sich Eigenkontrolle und Selbstmedikation vielfach bewährt. - Ob die telemetrische Übermittlung der gemessenen Werte, die bei Diabetikern und bei der Antikoagulation nicht erfolgt, bei der Hypertoniebehandlung einen Zusatznutzen bringt, ist fraglich und bedarf einer Überprüfung. In der Arbeit gibt es keine Hinweise, ob und welche therapeutischen Konsequenzen daraus folgten.

H. HolzGREVE =

\section{- R. J. McManus et al.}

Telemonitoring and self-management in the control of hypertension (TASMINH2): a randomised controlled trial. Lancet 376 (2010) $163-172$. 